\title{
Chemotherapy-related leiomyopathy: a suggested morphological explanation for the intestinal dysmotility affecting patients treated with anthracyclines
}

\author{
Karmaine A Millington ${ }^{1}$, Judy Mae Pascasio ${ }^{1}$, Gregory E Halligan ${ }^{2}$ and \\ Jean-Pierre de Chadarévian ${ }^{1}$ \\ ${ }^{1}$ Department of Pathology and Laboratory Medicine, Drexel University College of Medicine \\ and St Christopher's Hospital for Children, Philadelphia, PA, USA and ${ }^{2}$ Department of Pediatrics, \\ Section of Oncology, Drexel University College of Medicine and St Christopher's Hospital for Children, \\ Philadelphia, PA, USA
}

\begin{abstract}
Anthracycline, used in oncological chemotherapy, has one well-known side effect: cardiotoxicity. Another is abnormal intestinal motility such as constipation and ileus, the pathogenesis of which, to our knowledge, has not been morphologically investigated. We conducted a study in search of morphological evidence that might shed some light on the pathogenesis of the motility dysfunction. Autopsies performed between 2002 and 2007 were reviewed to select cases of children who had received anthracycline therapy for various neoplasms. The seven patients found had leukemias, lymphomas, or renal solid tumors. They all suffered from constipation or intestinal dysmotility, and no case of anthracyclin-treated neoplasia without the side effect was found in the files. Tissue samples from the heart, gastrointestinal tract, uterus, urinary bladder, and skeletal muscles were examined by light and electron microscopy. As described by others, the myocardium of all anthracyclinetreated patients showed loss of myofilaments, fibrosis, mitochondrial proliferation, and pools of accumulated Z-band material. In the gastrointestinal tract and other smooth muscle-endowed organs such as muscular blood vessels, bladder and uterus, the muscularis displayed hyalinization and disorganization, including loss of myofilaments and moderate-severe fibrosis. This study illustrates changes in the smooth muscle, and that of the gastrointestinal tracts and their vessels in particular, in patients treated with anthracycline, who had experienced motility dysfunction associated with their chemotherapy, suggesting that, in addition to the heart, anthracycline may also damage smooth muscle fibers and thus be instrumental in the pathogenesis of the side effects.

Modern Pathology (2012) 25, 289-294; doi:10.1038/modpathol.2011.115; published online 28 October 2011
\end{abstract}

Keywords: anthracycline; chemotherapy; constipation; intestinal motility; side effects

Anthracyclines are a class of antibiotics derived from Streptomyces species. The drug names in this class include daunorubicin (daunomycin, lyormal), doxorubicin (adriamycin), epirubicin (ellence), idarubicin (idamycin), valrubicin, aclarubicin, amrubicin, pirarubicin, and vorubicin. The anthracyclines have been used for more than three decades

Correspondence: Dr J-P de Chadarévian, MD, The Department of Pathology and Laboratory Medicine, St Christopher's Hospital for Children, 3601 A Street, Philadelphia, PA 19134, USA.

E-mail: j.dechadarevian@drexelmed.edu

Received 11 January 2011; revised 18 May 2011; accepted 31 May 2011; published online 28 October 2011 to treat a variety of malignancies including leukemias, lymphomas, carcinomas, and sarcomas. The known side effects range from mild gastrointestinal disturbances to myelosuppression and cardiovascular complications such as heart failure. Cerebrovascular complications, co-agulopathies, and even Raynaud's disease have been occasionally observed. ${ }^{1,2}$ The well-documented side effect which most often limits treatment is a life-threatening cardiomyopathy. ${ }^{2}$ Another side effect reported by the vast majority of patients is intestinal dysmotility including chronic constipation and episodes of ileus. The pathogenesis of this gastrointestinal disturbance is not clear and, to our knowledge, has 

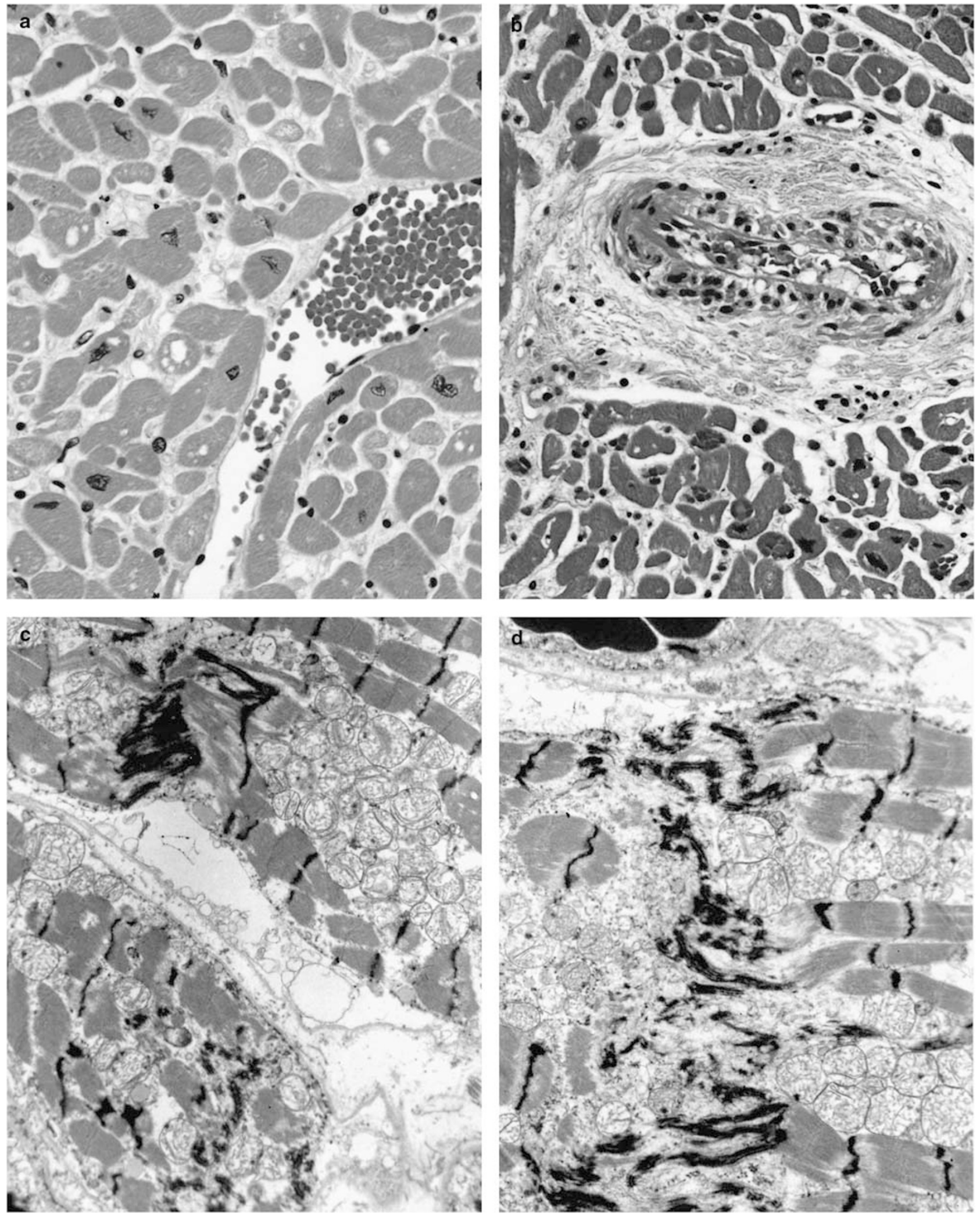

Figure 1 Cardiac pathology. (a) Histological appearance of the myocardium where vacuolated cells and boxcart nuclei can be seen. (b) A small branch of a coronary artery showing damage to the muscularis, essentially made of loss of smooth muscle fibers. (c and d) Ultrastructural illustration of the alterations affecting the myocardial cells in which mitochondrial proliferation, loss of myofibirls, and accumulation of pools of disorganized Z-band material can be seen. 
not been the subject of a morphological investigation. Therefore, we decided to conduct a study in search of morphological evidence that might shed some light on the pathogenesis of this side effect.

\section{Materials and methods}

Autopsies performed in our institution between 2002 and 2007 were reviewed to select cases of children who had received anthracycline therapy for various neoplasms. Anthracyclines used in these patients were in the form of daunomycin (daunorubicin), mitoxantrone, an anthracenedione related to anthracyclines, and adriamycin (doxorubicin).

\section{Clinical Data}

Seven cases who suffered of intestinal motility dysfunction were found, and no case of patient treated with anthracycline without experiencing intestinal motility problems was found in the files. The primary diagnoses included three acute myelogenous leukemias in patients aged 16, 18 years, and 21 months with trisomy 21 mosaicism; a 10-year-old T-cell lymphoma; a 21-year-old patient with spina bifida eventually necessitating renal transplantation complicated by a post-transplant large B-cell lymphoma, and two patients with renal solid tumors, aged 3 (Renal sarcoma) and 9 years (Wilms' tumor). Review of their clinical history revealed that they all suffered from chronic constipation, intestinal dilatation, or episodes of ileus, in addition to gastroparesis that was observed in one, the 3 yearold with the renal sarcoma. In all cases, dysmotility problems started a couple of weeks after the onset of chemotherapy and lasted till death.

\section{Pathology Materials and Methods}

Tissue blocks from the heart, gastrointestinal tract, bladder, uterus, and skeletal muscles were studied by light and electron microscopy. Controls were archival tissue blocks from autopsies of patients who never had been diagnosed with a neoplasm, never received anthracycline, and had no history of chronic constipation or intestinal dysmotility. Formalin-fixed, paraffin-embedded tissue sections were stained with hematoxylin-phloxine-saffron (HPS), Gomori Trichrome, phosphotungstic acidhematoxylin (PTAH), and periodic acid-Schiff (PAS). Samples fixed in 3\% glutaraldehyde were epon-embedded and processed conventionally for transmission electron microscopy, using a Jeol electron microscope, model JEM-1011.

\section{Results}

Based on review of autopsy data, it appeared that, save for one patient, the 21 year-old with post-transplant large B-cell lymphoma, all remaining six treated patients had cardiomegaly, and four of them had pronounced ventricular dilatation.
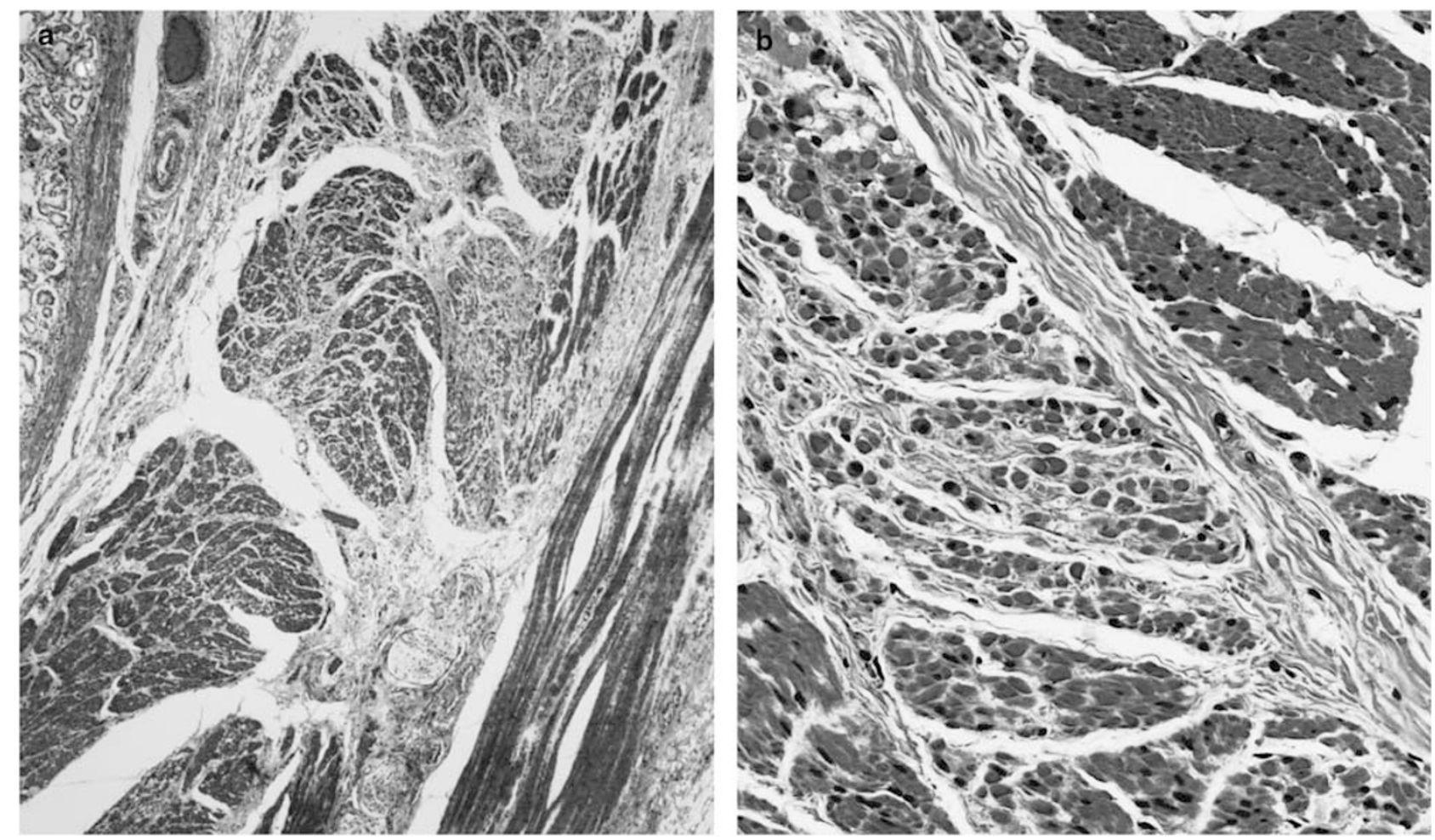

Figure 2 Histological illustration of the fibrosis and loss of smooth muscle cells in (a): the stomach, and (b): the urinary bladder, as demonstrated by the Masson trichrome stain. 

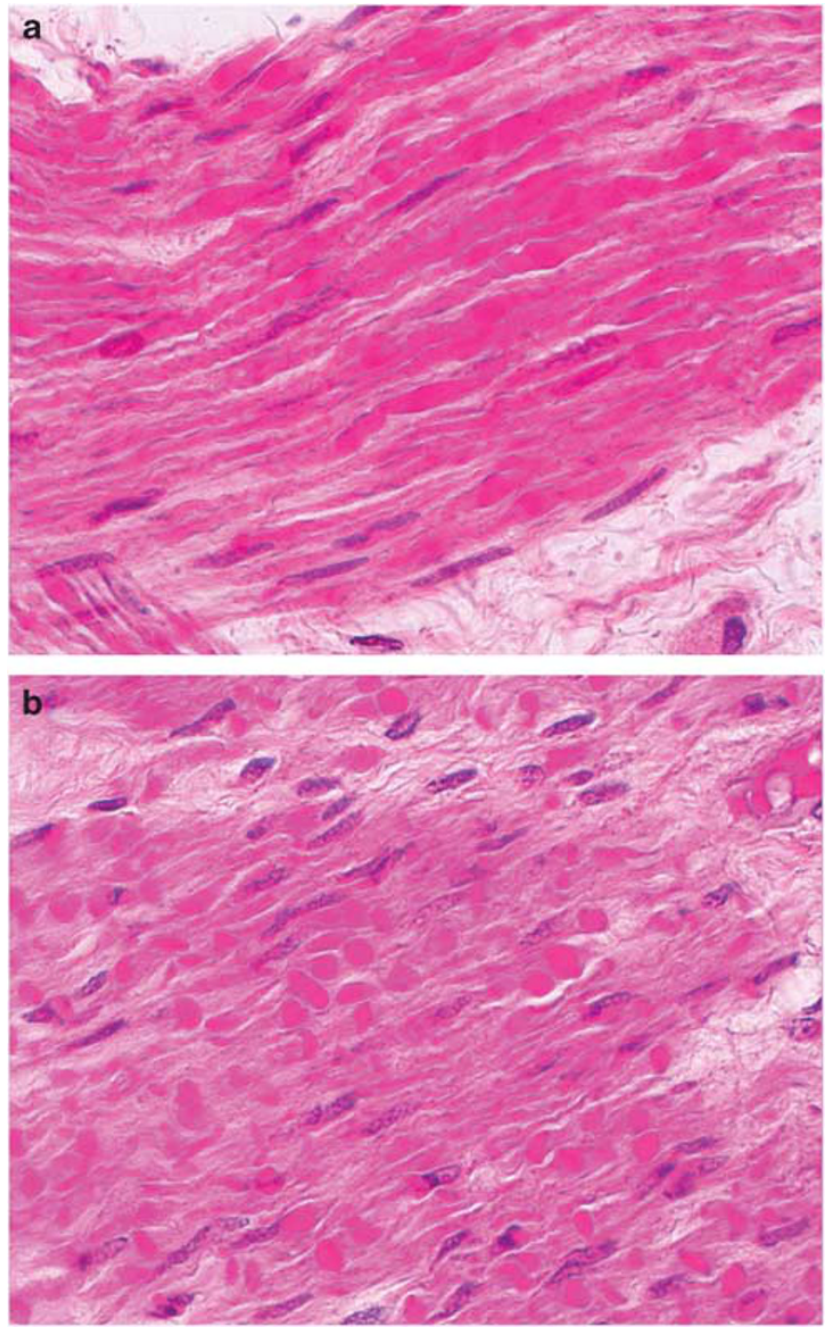

Figure 3 Histological illustration of the smooth muscle damage as seen in (a) longitudinal section reminiscent of the astrocytic Rosenthal fibers, and (b) cross section, both from intestinal samples stained with HPS.

The heart weights at autopsy were $75-100 \%$ above normal for patients' age. However, histological sections of the myocardium of all seven patients showed hypertrophic muscle fibers with boxcarshaped nuclei and variable degrees of interstitial fibrosis. There was also cytoplasmic vacuolization in some of the fibers, which was variable in shape, size, and number per affected myocytes (Figure 1a). Also noted at the level of the myocardium were vascular alterations made of loss of smooth muscle fibers, when compared with controls (Figure 1b). This type of change was not confined to the coronary branches, but could be seen in most examined organs, such as bladder and uterus. Ultrastructural examination of the heart showed fibrosis, mitochondrial proliferation, and loss of myofilaments leading to haphazardly arranged aggregates of Z-band material (Figures 1c and d).

Throughout the gastrointestinal tract of the treated patients, the muscularis propria and muscularis mucosae displayed fibrosis usually associated with dropout or atrophy of muscle fibers (Figures 2a and b). Examination of the smooth muscle fibers at high magnifications showed vacuolization of the cytoplasm seen in some sections, and widespread hyalinization of the fibers observed in all patients (Figures 3a and b). Ultrastructurally, the hyalinized muscle fibers resembled bags of parallel intermediate-looking filaments with haphazardly distributed 'focal densities' (Figure 4a), sometimes aggregated and confined to the periphery of the fiber (Figure 4b). Those fibers also appeared almost totally devoid of organelles, such as endoplasmic reticulum and mitochondria. The changes were not identical in extent among patients, but were relatively consistent for a given individual. Similar changes were noted in the uterus and bladder (Figure 2b). Innervation of the gastrointestinal tract and other sites examined did not show any recognizable abnormality.

The alterations histologically and ultrastructurally observed in the treated group were not seen in the controls, but also surprisingly, no lesion could be detected in the skeletal muscle of the treated patients.

\section{Discussion}

In addition to myocardial injury, this study demonstrates morphological abnormalities in the smooth muscle, particularly in the gastrointestinal tract, muscular intestinal vessels, urinary bladder, and uterus of patients treated with anthracycline and who had experienced problems related to intestinal dysmotility, such as chronic constipation and ileus associated with the onset of their chemotherapy and lasted throughout the duration of the treatment. This leads us to believe that the chemotherapy was most likely instrumental in the pathogenesis of the dysmotility, and, based on the data previously established at the level of the myocardium, the most likely culprit seems to be anthracycline, although the role of other agents the patients received can be debated, as none was treated with only anthracycline, and, albeit they received a vast array of adjuvant chemotherapeutic agents (too many to be listed individually) depending on the diagnosis-related therapeutic protocol, the only constant in the management of the seven cases was a form of anthracycline (mitoxantrone, daunomycin, or adriamycin).

We are aware of very few significant studies looking into the mechanisms of the chemotherapyrelated intestinal smooth muscle injury. Thus, until the results of further investigations of the subject emerge, it seems reasonable to hypothesize that many, if not most of the mechanisms invoked at the level of the myocardium may be operative at the smooth muscle level. 

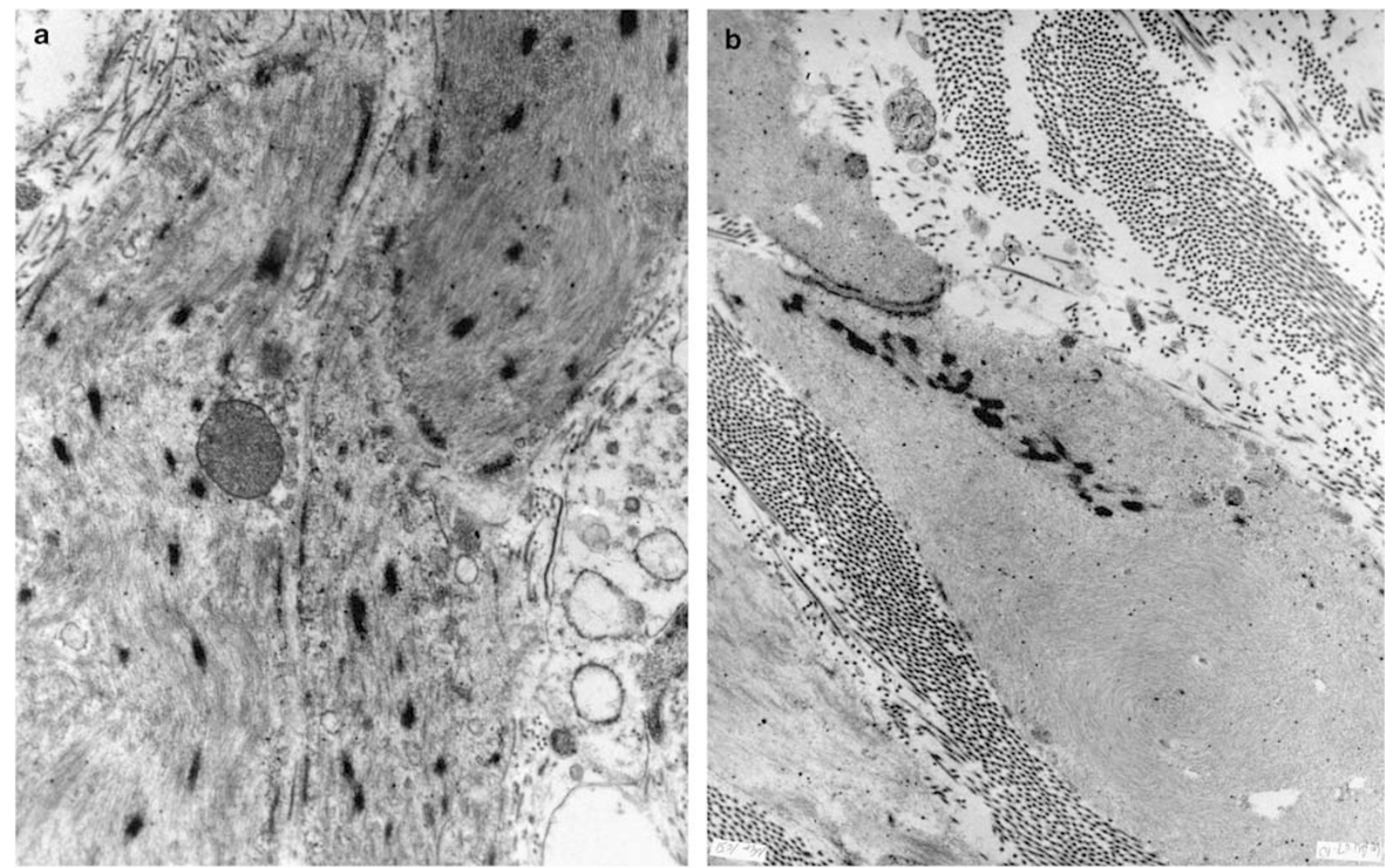

Figure 4 Ultrastructural appearance of the injured intestinal smooth muscle fibers. (a) Loss of myofibrils and pooling of haphazardly arranged 'densities'. (b) More severe damage reducing the smooth muscle fiber to a bag of intermediate filaments with some densities pushed to the periphery and absence of organelles.

Anthracycline-induced cardiotoxicity is thought to be multifactorial, including the selective inhibition of cardiac muscle gene expression for alphaactin, troponin, myosin light-chain $\mathrm{Z}$ and the $\mathrm{M}$ isoforms of creatine kinase in vivo, the free radicalmediated myocyte damage, myocyte damage from calcium overload disturbances in myocardial adrenergic function, release of vasoactive amines, cellular toxicity from metabolites and anthracyclines, and pro-inflammatory cytokines. ${ }^{3}$ It has been suggested that the cardiotoxicity of anthracyclines occurs by the suppressed transcription of sarcomeric proteins, as the planar structure of anthracyclines allows for intercalation between paired bases in the DNA helix. This non-covalent interaction would be strong enough to inhibit DNA and RNA synthesis, and thereby allows accumulation of DNA mutations, particularly in the mitochondria. One early event in the breakdown of sarcomeres after anthracycline exposure is also the degradation of titin, the integrity of which is critical for the dynamic regulation of contractile function via the Frank-Starling mechanism of length-dependent changes in the sensitivity of actin-myosin cross-bridges to calcium. The loss of intact titin has been demonstrated in ischemic and failing human hearts, and anthracycline treatment of cardiac myocytes has been shown to result in accelerated titin degradation. ${ }^{4}$

The mechanisms of vascular injury have been also extensively investigated, and the findings reported may apply to the injury observed in the intestinal vessels. It has been found that aclarubicin, an anthracycline, inhibits nitric oxide production in rat aortic smooth muscle cells. ${ }^{1}$ Reported anthracyclines actions on vessels have been also attributed to persistent release of noradrenaline from sympathetic nerve terminals, resulting in a gradual increase in the resting tension of the vessel. ${ }^{1}$ Downregulation of the $\alpha_{1 \mathrm{~A}}$-adrenoceptor expression was predominant at a lower concentration of doxorubicin, and apoptosis and necrosis were seen at higher concentrations. Oxidative stress and impairment of mitochondrial function are associated with alterations in cellular $\mathrm{Ca}^{2+}$ homeostasis and gene expression, and it has been suggested that they may mediate the vascular noxious action of anthracyclines. Direct interference of anthracyclines with membrane proteins and lipids must be also considered as possible mechanism by which the drug affects the vessels. Modulation of endothelial nitric oxide (NO) generation by anthracyclines is another mechanism underlying side effects of anthracyclines. Yet, endothelial effects of anthracyclines 
are not only confined to NO generation. Experimentally, a low concentration of daunorubicin significantly attenuates acetylcholine-induced relaxation of the rat aorta without affecting the tissue c-GMP level in response to acetylcholine. The effects are abolished by $\mathrm{K}+$ channel blockade and tissue depolarization with tetraethylammonium. These results suggest that daunorubicin may inhibit relaxation mediated by endothelium-dependent hyperpolarizing factor(s) (EDHF), most likely due to depolarization of the vascular smooth muscle cells. Doxorubicin was also found to cause apopotosis of both cardiomyocytes and endothelial cells. Intracellular $\mathrm{Ca} 2+$ signals appear to have a pivotal role in the pro-apoptotic action of anthracyclines, as induction of programmed cell death by doxorubicin was prevented by chelation of intracellular $\mathrm{Ca} 2+.{ }^{1}$

The absence of detectable damage to the skeletal muscle in our patients is not easy to explain. It may be related to physiological differences between muscle types, a subject that would benefit from further investigation.

In summary, this pathological study of the organs of patients who had experienced intestinal dysfunction associated with their chemotherapy demonstrates morphological abnormalities in the myocardium, as well as a leiomyopathy particularly affecting the gastrointestinal tract and intestinal muscular vessels. Albeit the subjects evaluated had been treated with different chemotherapeutic regimens comprising multiple agents depending on their type of malignancy, those regimens only had anthracycline in common, and based on the data previously established at the level of the myocardium, the most likely culprit in the pathogenesis of the leiomyopathy described seems to be anthracycline.

\section{Disclosure/conflict of interest}

The authors declare no conflict of interest.

\section{References}

1 Wakabayashi I, Groschner K. Vascular actions of anthracycline antibiotics. Curr Med Chem 2003;10: 427-436.

2 Mordente A, Meucci E, Martorana GE, et al. Human heart cytosolic reductases and anthracycline cardiotoxicity. IUBMB Life 2001;52:83-88.

3 Shan K, Lincoff M, Young JB. Anthracycline-induced cardiotoxicity. Ann Intern Med 1996;125:47-58.

4 Chen B, Peng X, Pentassuglia L, et al. Molecular and cellular mechanisms of anthracycline cardiotoxicity. Cardiovasc Toxicol 2007;7:114-121. 